\title{
A micro-SQUID with dispersive readout for magnetic scanning microscopy $\odot \odot$
}

Cite as: Appl. Phys. Lett. 112, 252601 (2018); https://doi.org/10.1063/1.5030489

Submitted: 22 March 2018. Accepted: 16 May 2018 . Published Online: 20 June 2018

(D) F. Foroughi, J.-M. Mol, T. Müller, (D) J. R. Kirtley, K. A. Moler, and H. Bluhm

\section{COLLECTIONS}

F This paper was selected as Featured

SCI This paper was selected as Scilight
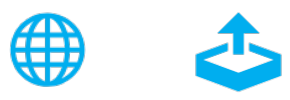

\section{ARTICLES YOU MAY BE INTERESTED IN}

Scanning SQUID susceptometers with sub-micron spatial resolution

Review of Scientific Instruments 87, 093702 (2016); https://doi.org/10.1063/1.4961982

Advances in micro-SQUID design could detect and manipulate single electrons

Scilight 2018, 250006 (2018); https://doi.org/10.1063/1.5044741

Scanning SQUID sampler with 40-ps time resolution

Review of Scientific Instruments 88, 083703 (2017); https://doi.org/10.1063/1.4986525

\section{Challenge us.}

What are your needs for periodic signal detection?

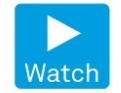

Zurich

- Instruments

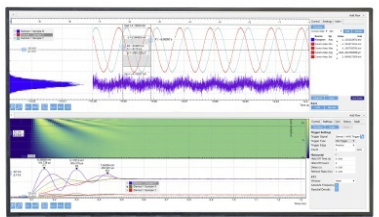

(0 


\title{
A micro-SQUID with dispersive readout for magnetic scanning microscopy
}

\author{
F. Foroughi, ${ }^{1, a)}$ J.-M. Mol, ${ }^{1, b)}$ T. Müller, ${ }^{1, c)}$ J. R. Kirtley, ${ }^{2}$ K. A. Moler, ${ }^{2}$ and H. Bluhm ${ }^{1,3}$ \\ ${ }^{1}$ 2nd Institute of Physics C, Quantum Technology Group, RWTH Aachen University, D-52074 Aachen, \\ Germany \\ ${ }^{2}$ Center for Probing the Nanoscale, Stanford University, Stanford, California 94305, USA \\ ${ }^{3}$ JARA-FIT Institute Quantum Information, RWTH Aachen University and Forschungszentrum Jülich GmbH, \\ D-52425 Jülich, Germany
}

(Received 22 March 2018; accepted 16 May 2018; published online 20 June 2018)

\begin{abstract}
We have designed and characterized a micro-SQUID with dispersive readout for use in low temperature scanning probe microscopy systems. The design features a capacitively shunted RF SQUID with a tunable resonance frequency from 5 to $12 \mathrm{GHz}$, micrometer spatial resolution, and integrated superconducting coils for local application of magnetic fields. The SQUID is operated as a nonlinear oscillator with a flux- and power-dependent resonance frequency. Measurements for device characterization and noise benchmarking were carried out at $4 \mathrm{~K}$. The measured flux noise above $10 \mathrm{kHz}$ at $4 \mathrm{~K}$ is $80 \mathrm{n} \Phi_{0} \mathrm{~Hz}^{-1 / 2}$ at a bandwidth of $200 \mathrm{MHz}$. Estimations suggest that one can benefit from parametric gain based on inherent nonlinearity of the Josephson junction and reduce the flux noise to $30 \mathrm{n} \Phi_{0} \mathrm{~Hz}^{-1 / 2}$ at $100 \mathrm{mK}$, which corresponds to $10.6 \mu_{B} \mathrm{~Hz}^{-1 / 2}$ for a magnetic moment located at the center of the pickup loop. Published by AIP Publishing.

https://doi.org/10.1063/1.5030489
\end{abstract}

Measuring the magnetic response of mesoscopic samples or mapping it vs. position for extended systems is effective methods for revealing the fundamental quantum properties of condensed matter systems. Over the past few decades, many advanced magnetic imaging schemes have been developed, including magnetic force microscopy, ${ }^{1}$ scanning Hall probe microscopy, ${ }^{2}$ superconducting interference devices (SQUIDs), ${ }^{3,4}$ and NV center based magnetometry. ${ }^{5}$ The high sensitivity, low back action, and low power dissipation of SQUIDs make them attractive for many types of low temperature experiments. One classic example is the central role of scanning SQUID microscopy in tests of pairing symmetry of high- $\mathrm{T}_{\mathrm{c}}$ cuprate superconductors. ${ }^{6}$ Other prominent applications are the thermodynamic characteristics of persistent currents in normal metal rings ${ }^{7}$ and proof of edge states in topological insulators in the quantum spin Hall regime. ${ }^{8}$

A key performance for a SQUID is its flux sensitivity. Parametric amplification has been harnessed in nanoSQUIDs based on aluminum junctions ${ }^{9,10}$ in order to reduce the flux noise down to $30 \mathrm{n} \Phi_{0} \mathrm{~Hz}^{-1 / 2}$ with a bandwidth exceeding $60 \mathrm{MHz} .^{10}$ Recent studies on parametric amplifiers and dispersive readout of superconducting qubits also harness the nonlinearity of the Josephson junction to boost sensitivity. ${ }^{11-13}$

In scanning SQUID microscopy, signal sources often behave dipole-like, such as superconducting vortices in mesoscopic dots or currents in mesoscopic rings. Smaller SQUIDs provide better coupling to smaller samples which leads to higher spin sensitivity ${ }^{3,14}$ as the magnetic field of a dipole decreases with $1 / r^{3}$. Various approaches have been

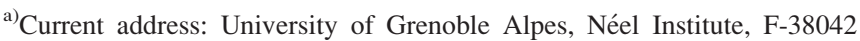
Grenoble, France.

b) Electronic mail: mol@physik.rwth-aachen.de.

c) Current address: Max-Planck Institute of Microstructure Physics, Weinberg 2, D-06120 Halle, Germany.
}

pursued to reduce the size. The smallest nano-SQUID to date has been fabricated by evaporating $\mathrm{Nb}$ or $\mathrm{Pb}^{15}$ onto the apex of a sharp quartz tip reaching a loop diameter of below nm and a spin sensitivity of $0.38 \mu_{\mathrm{B}} \mathrm{Hz}^{-1 / 2}$ at tens of $\mathrm{kHz}$ bandwidth. Compared to such size-optimized devices, microSQUIDs fabricated using a standard $\mathrm{Nb}$ technique have the advantage of on-chip field coils and modulation coils, ${ }^{14}$ and can be operated at $4 \mathrm{~K}$ allowing for a wide range of applications. However, these come at the cost of larger pick-up loops of a few microns and a resulting poorer spin sensitivity of $200 \mu_{\mathrm{B}} \mathrm{Hz}^{-1 / 2}$ for a magnetic moment located at the center of the pickup loop.

The limitation of large pickup loops was previously only overcome by devices with pickup loops defined by the focused-ion-beam, ${ }^{16}$ but has recently also been achieved by improvements in lithography and shown to offer better spatial resolution as well. ${ }^{17}$ Here, we present a scanning microSQUID which is based on the same fabrication technology incorporating another major advancement. Our design exploits the parametric amplification based on nonlinearities of the SQUID to boost sensitivity. The combination of a smaller pickup loop and parametric amplification of the signal leads to better spin sensitivity $\left(10.6 \mu_{\mathrm{B}} \mathrm{Hz}^{-1 / 2}\right)$ and higher bandwidth $(200 \mathrm{MHz})$ compared to traditional DC microSQUIDs in which the flux sensitivity is mainly limited by internal dissipation.

Our devices were fabricated at Hypres Inc. using planarized $\mathrm{Nb} / \mathrm{AlO}_{\mathrm{x}} / \mathrm{Nb}$ trilayer Josephson junction technology, including two planarized $\mathrm{Nb}$ layers with approximately $0.8 \mu \mathrm{m}$ minimum feature size. The SQUID consists of a superconducting ring shunted by one Josephson junction with a designed critical current of $20 \mu \mathrm{A}$ [Fig. 1(a)]. With this geometry, the SQUID is gradiometric, i.e., the effect of any homogeneous background magnetic field approximately cancels out. The smallest pickup loops implemented have an inner (outer) diameter of $1 \mu \mathrm{m}(2 \mu \mathrm{m})$. The superconducting 


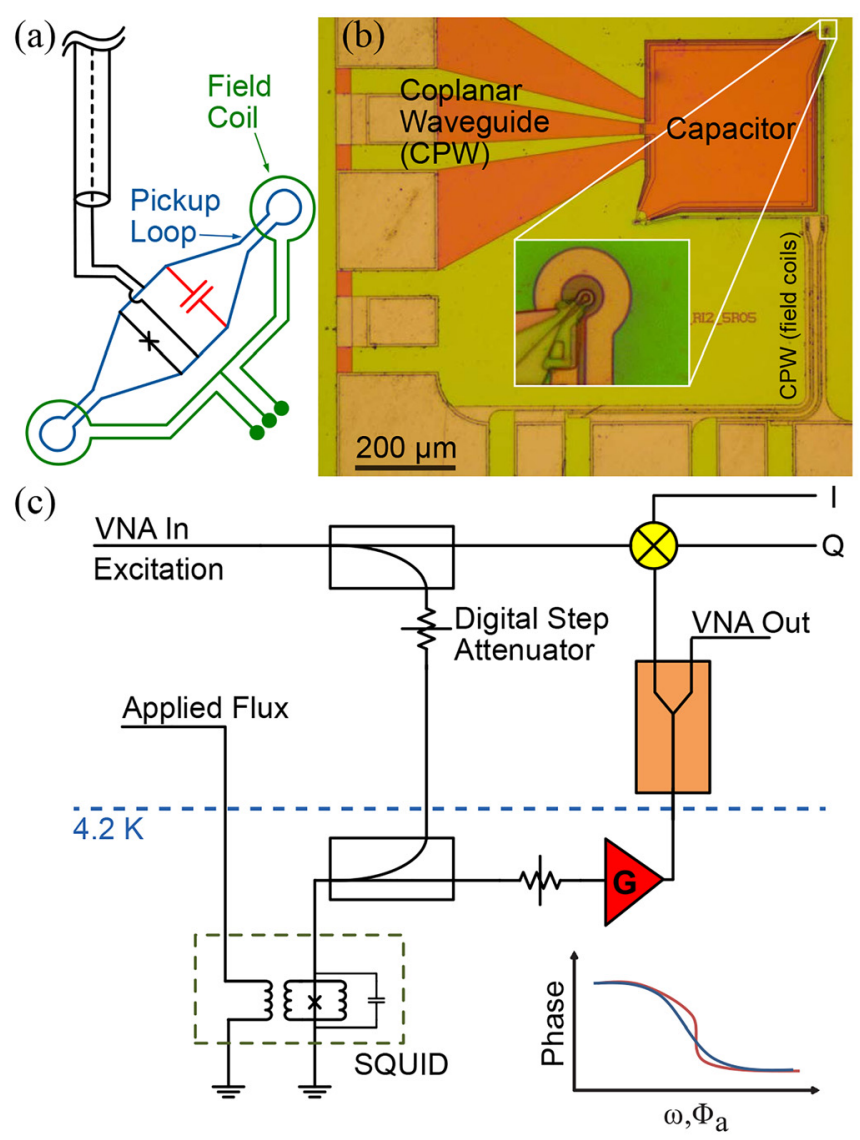

FIG. 1. (a) Schematic circuit diagram of the SQUID. The geometry is gradiometric and cancels out any homogeneous background magnetic field. Two field coils with a center-tab allow applying magnetic fields to the sample while the total flux threading the SQUID is unchanged. (b) Optical image of the fabricated chip. (c) Simplified schematic of the read-out setup. The inset shows the effect of increased power (red line) on the reflected phase of the RF signal as a function of frequency (or applied magnetic flux $\Phi_{a}$ ).

loop is shunted by an on-chip parallel-plate capacitor with amorphous silicon dioxide as dielectric which was designed to have a capacitance of $80 \mathrm{pF}$. The superconducting loop and the capacitor form a resonator with a flux dependent resonance frequency from 5 to $12 \mathrm{GHz}$. The pickup loops are placed on opposite corners of the parallel-plate capacitor at a distance of about $450 \mu \mathrm{m}$ so that one loop can be located in close proximity to the sample while the other is kept far away from it. External fields can be applied to the sample by local single-turn field coils situated around each pickup loop. The field coils are fully integrated into the chip layout and can also be used to bias the SQUID at its most sensitive point. Compared to using additional modulation coils, ${ }^{14}$ this approach has the advantage of reducing the total device inductance, which leads to a higher sensitivity. ${ }^{18}$ In a scanning microscope, the field coil at the rear end (away from the sample) can be used to bias the SQUID or operate it in flux feedback such that resulting stray fields will not act on the sample, apart from a weak screening current through the pickup loop. The effective diameter of each coil is $7.5 \mu \mathrm{m}$, resulting in a mutual inductance of about $0.34 \Phi_{0} / \mathrm{mA}$ (for the smallest pickup loop). The SQUID resonator is connected by an on-chip $Z_{0}=50 \Omega$ coplanar waveguide (CPW) transmission line to the bonding pads. The two field coils are connected by two smaller CPWs to the bottom edge bonding pads [Fig. 1(c)], ending in two parallel-stripline waveguides running alongside the edges of the capacitor towards the respective field coils. Based on estimations, it should thus be possible to apply high frequency signals of at least several $\mathrm{GHz}$ to SQUID and sample.

As readout, we adopted a dispersive approach. ${ }^{9}$ The SQUID is operated as a nonlinear oscillator with a flux- and power-dependent resonance frequency. A continuously applied microwave excitation is reflected by the SQUID with a flux dependent phase shift which can be detected using standard homodyne detection. Figure 1(c) shows a simplified schematic of this scheme. To attenuate room temperature electronic and thermal noise down to the level of Johnson noise at $4 \mathrm{~K}, \mathrm{RF}$ lines are attenuated at low temperature. A directional coupler is used to couple the excitation to the SQUID and guide the reflected signal to a semiconductor cryogenic amplifier. The amplifier has a bandwidth of $1-12 \mathrm{GHz}$ so that it does not limit the SQUID's operation. The measured noise temperature of the amplifier is about $5 \mathrm{~K}$ between 3 and $6 \mathrm{GHz}$. The signal is further amplified by several room temperature amplifiers before being sent to the vector network analyzer (VNA) or to a mixer, where the phase of the reflected signal can be read out. While the I and Q outputs are most convenient for high bandwidth measurements, we characterize the SQUID using a VNA to measure the reflection coefficient $(\Gamma)$ of the SQUID as a function of applied magnetic flux and RF power.

An external magnetic flux applied to one pickup loop will induce a circulating current in the SQUID resonator and its Josephson junction which changes the Josephson inductance. Due to the symmetry of the parallel circuit, part of the circulating current is shunted through the other pickup loop inductance and reduces the effect of the external flux by a factor of two [see Fig. 1(a)]. Thus, the resonance frequency of the tank circuit and hence the mixer output voltage is a periodic function of the applied external magnetic flux $\left(\Phi_{a}\right)$ with a periodicity of $2 \Phi_{0}$ [see Fig. 2(a)]. Fitting a simple model of a LC tank-circuit shunted by a Josephson junction, we extracted a critical current of $23 \mu \mathrm{A}$, a loop inductance of $19 \mathrm{pH}$, and a capacitance of $30 \mathrm{pF}$. At finite static bias, especially near $\Phi_{a} \approx \Phi_{0}$, the resonance frequency is very sensitive to the applied flux, so the highest sensitivity is achieved by biasing the SQUID on resonance at a point with the largest slope. The transduction factor $d V / d \Phi=G_{\text {tot }} v_{\text {in }}(d \Gamma / d \Phi)$ represents the change in the reflected microwave signal at the mixer or VNA input in response to the change in the external magnetic flux, where $v_{\text {in }}$ is the amplitude of the excitation applied to the SQUID and $G_{\text {tot }}$ is the total gain of the amplifiers before the mixer and its conversion gain. In the linear regime, $\Gamma$ is independent of the excitation and hence the flux sensitivity can be improved by both increasing the excitation amplitude and biasing the SQUID at higher $|d \Gamma / d \Phi|$.

The analysis discussed above assumes that the excitation is small enough $(<-95 \mathrm{dBm})$ for the SQUID to behave as a linear inductor. When increasing the power, nonlinear effects arising from the sinusoidal phase-current characteristic of the Josephson junction become important. With moderate excitation, one can harness inherent nonlinearities in the SQUID to enhance the sensitivity [inset of Fig. 1(c)]. 
(a)

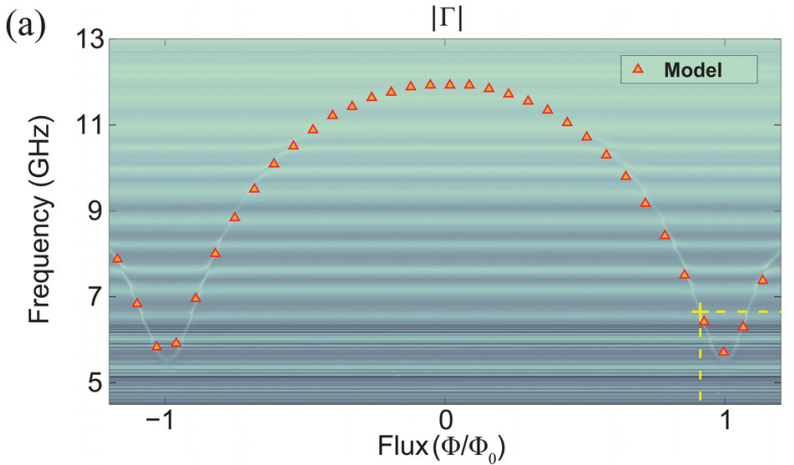

(b)

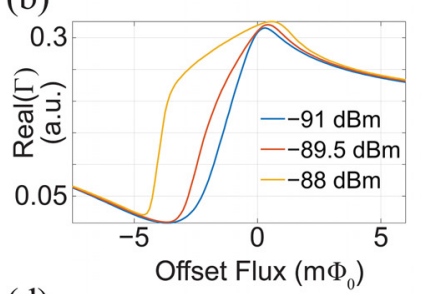

(d) $\quad|\mathrm{dV} / \mathrm{d} \Phi|\left(\mathrm{mV} / \Phi_{0}\right)$
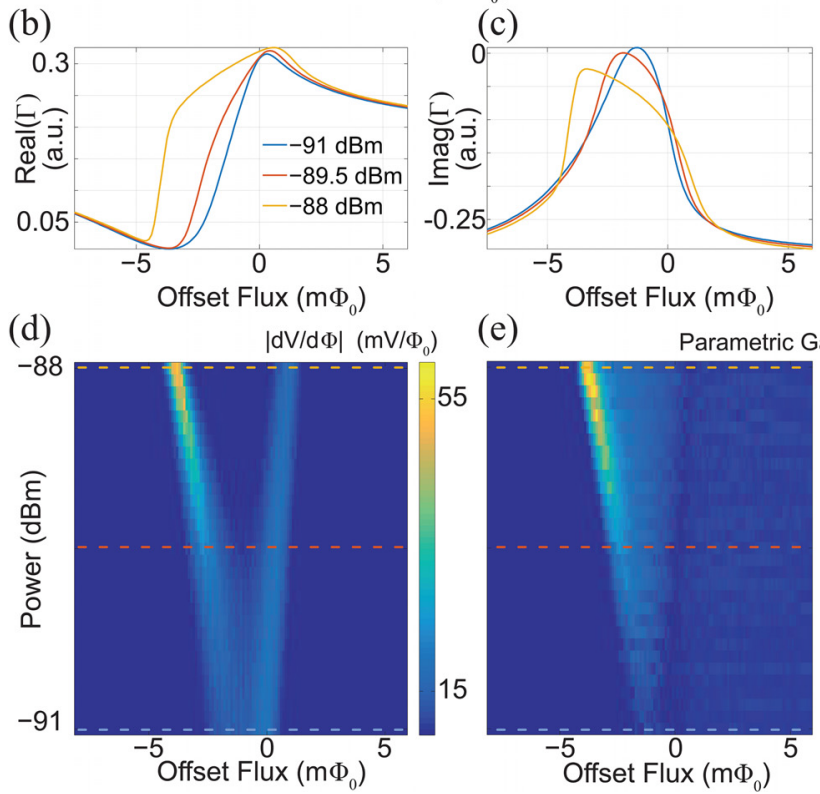

(e)

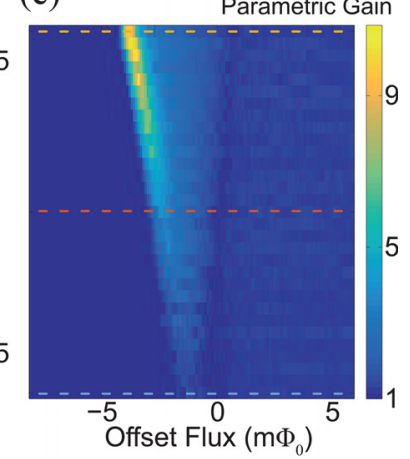

FIG. 2. (a) Dependence of resonance frequency on the applied magnetic field by one of the field coils and comparison with simulation (triangles). The resonance frequency is a periodic function of the external field with a periodicity of $2 \Phi_{0}$ (see the text). The horizontal lines visible are caused by resonances in the system due to imperfect impedance matching. (b) Real and (c) imaginary part of the reflected microwave signal at different powers measured by a VNA. Traces are recorded at a coarse flux bias of $\Phi_{a}=0.91$ $\Phi_{0}$ and $6.66 \mathrm{GHz}$ driving frequency [yellow cross in (a)], while fine sweeping with an additional flux bias. Nonlinearities lead to a typical bending of the resonance curve at high driving powers. (d) Transduction factor estimation based on excitation power and external bias sweeps. The bending results in a splitting of flux sensitive areas, with an enhanced transduction at the steeper side. (e) Estimated parametric gain $|G|$; only the left branch shows parametric amplification. The data in (d) and (e) were computed by numerical differentiation of the reflected signal. The off-resonant gain was normalized to one to compensate for any microwave signal losses or reflections in the system.

However, nonlinearities eventually lead to bifurcation which limits the maximum power level that can be applied to the SQUID. Hence, a crucial factor for optimum noise performance while avoiding bistable operating points is a careful choice of the excitation power and flux bias. When the power is low enough, experimentally, we find the lowest-noise operating points near $\Phi_{a}=0.91 \Phi_{0}$, which is in the region where the resonance frequency is most sensitive to the applied flux [Fig. 2(a)]. To further investigate the noise performance of the SQUID, two dimensional sweeps in $\Phi_{a}$ (small offset flux added to $\Phi_{a}=0.91 \Phi_{0}$ ) and applied power at a constant frequency of $6.66 \mathrm{GHz}$ were carried out. Figure 2(d) shows the estimated transduction factor. At low powers, there is a single sensitive region at resonance. At higher

power, this flux sensitive region splits up into two branches; the left branch with an enhanced transduction factor and the right one without any enhancement. This behavior arises phenomenologically due to bending of the resonance at high driving amplitudes [see Figs. 2(b) and 2(c)]. ${ }^{12}$ The distortion of the resonance curve on the left branch leads to a faster change of the reflected phase as a function of the applied flux (increase in $|d \Gamma / d \Phi|$ ) and thus improves the transduction factor, as illustrated in the inset of Fig. 1(c). This behavior is representative for a broad range of operating frequencies, with the exception of very low and very high frequencies. Approaching high frequencies (close to even flux quanta), the transduction factor decreases and the SQUID becomes insensitive to flux. At lower frequencies (odd flux quanta), the power at which the nonlinearities become dominant decreases significantly, limiting the resulting signal-to-noise ratio.

While nonlinearities result in an enhancement of the transduction factor, the spectral noise density of the SQUID $S_{\Phi}^{1 / 2}=S_{V}^{1 / 2} /|d V / d \Phi|$ is also affected by parametric amplification of noise on the excitation line. To estimate the total noise level, two contributions have to be taken into account. First, the semiconductor cryogenic amplifier adds its own noise to the reflected signal. Second, noise on the drive line is amplified by the SQUID with parametric voltage gain $G=\Gamma^{-1}\left(d v_{\text {ref }} / d v_{\text {in }}\right)=1+v_{\text {in }} \Gamma^{-1}\left(d \Gamma / d v_{\text {in }}\right)$, where $v_{\text {ref }}$ $=\Gamma v_{\text {in }}$ is the complex voltage amplitude of the reflected signal at the SQUID. The above expression assumes conservation of energy so that changes in $v_{\text {in }}$ can only affect the phase of $v_{\text {ref. }}$ An estimate of $|G|$ based on the dependence of the measured reflected signal on the excitation power is shown in Fig. 2(e). This parametric amplification amplifies the power of signals in phase with the excitation by $|G|^{2}$. The spectral noise density for the sensitive quadrature is thus $S_{V}^{1 / 2}=\sqrt{k_{\mathrm{B}}\left(T_{\mathrm{amp}}+|G|^{2} T_{\text {eff }}\right) Z_{0} / 2}$, where $k_{\mathrm{B}}$ is the Boltzmann constant, $T_{\mathrm{amp}}$ is the equivalent input noise of the cryogenic amplifier, and $T_{\text {eff }}$ is the effective Johnson noise temperature considering the effect of quantum noise at the excitation frequency and the base temperature $\left(k_{\mathrm{B}} T_{\text {eff }}=\hbar \omega \operatorname{coth}\left(\hbar \omega / k_{\mathrm{B}} T_{\text {base }}\right)\right)$. At $4 \mathrm{~K}$, the thermal Johnson noise is close to the noise associated with the first stage semiconductor microwave amplifier $(5 \mathrm{~K})$, requiring only very small parametric gain in the SQUID for the amplified noise to exceed the noise from the semiconductor amplifier. However, we expect that at lower temperature, a stronger excitation can be applied to the SQUID to provide higher parametric gain in order to reduce the effect of amplifier noise before noise amplification becomes dominant. Figures 3(a) and 3(b) show the noise performance estimated from the data in Figs. 2(b) and 2(c) using the above relations at $4 \mathrm{~K}$ and $100 \mathrm{mK}$. In the non-amplified branch (right branch), the SQUID does not show enhancement in flux sensitivity. This also coincides with zero parametric gain and hence no noise rise. At $100 \mathrm{mK}$, biasing the SQUID on the parametrically amplified branch, reduces the overall noise temperature of the amplification chain, therefore the noise performance is enhanced. In this branch, the complication arises that the reduction of the transduction coefficient at higher parametric 

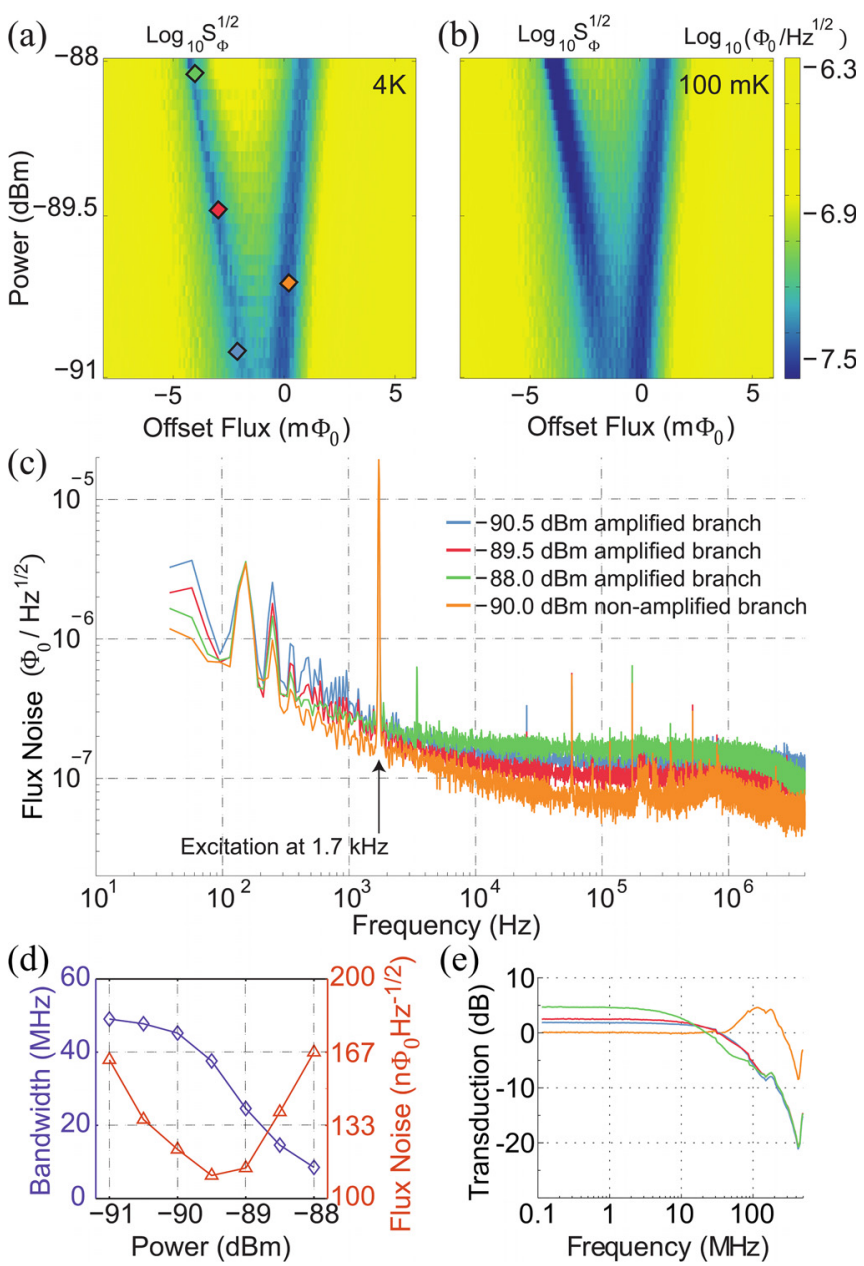

FIG. 3. (a) and (b) Estimated flux noise based on the data from Fig. 2 and the noise model described in the text for $T=4 \mathrm{~K}$, and $T=100 \mathrm{mK}$. (c) Measured flux noise at $4 \mathrm{~K}$ and at different bias points on flux noise estimation. In order to simultaneously measure the transduction coefficient, an ac signal with an amplitude of $20 \mu \Phi_{0}$ and frequency of $1.7 \mathrm{kHz}$ was applied to one of the field coils and the voltage noise was obtained by averaging the noise floor between $50 \mathrm{kHz}$ and $100 \mathrm{kHz}$. (d) Dependence of the bandwidth and flux noise on excitation power on the parametrically amplified branch. (e) Corresponding transduction of excitation vs. frequency for the different bias points shown in (a) and (c).

gain eventually degrades the noise performance of the device, in particular, at $4 \mathrm{~K}$ [Fig. 3(e)]. This effect is attributed to phase sensitive amplification in parametric amplifiers ${ }^{10}$ where noise is amplified more than the transduction because the phase of the flux signal does not coincide with the amplified quadrature.

To verify the noise model, we directly measured the noise at different points on both non-amplified and parametrically amplified branches [Fig. 3(c)]. At each different bias point, we measured the spectral power density of the mixer output. The flux noise was estimated by dividing the voltage spectral density by the transduction coefficient computed from the area under the excitation peak.

At $4 \mathrm{~K}$, the best operating points lie on the nonamplified branch where parametric gain is negligible. We obtained an effective flux noise of $80 \mathrm{n} \Phi_{0} \mathrm{~Hz}^{-1 / 2}$. This agrees well with an estimated best noise performance of $\sim 76$ $\mathrm{n} \Phi_{0} \mathrm{~Hz}^{-1 / 2}$ [Fig. 3(a)]. Nevertheless, the estimate in Fig. 3(b) predicts that at a base temperature below $\sim 300 \mathrm{mK}$ where quantum noise dominates, one can harness the parametric gain in the parametrically amplified branch and reduce the flux noise to $30 \mathrm{n} \Phi_{0} \mathrm{~Hz}^{-1 / 2}$. According to Tesche and Clarke, ${ }^{19}$ a typical DC SQUID with two non-hysteretic, resistively shunted tunnel junctions, in the limit of $\beta \approx 1$, and with inductance and junction critical current density equivalent to our device would perform at a flux noise of $S_{\Phi}^{1 / 2}=8 k_{\mathrm{B}} T L \sqrt{\pi L C} \approx 292 \mathrm{n}_{0} \mathrm{~Hz}^{-1 / 2}$, ultimately quantum limited by $S_{\Phi}^{1 / 2}=h L=79 \mathrm{n}_{0} \mathrm{~Hz}^{-1 / 2}$ at dilution refrigerator temperatures. We attribute the improved noise performance of our devices to the advantages of dispersive readout.

Note that the bandwidth decreases as the parametric gain increases. The bandwidth was determined by sweeping the ac signal frequency at the field coil. For the nonamplified branch, it is about $200 \mathrm{MHz}$ and independent of the applied power. This value lies close to an estimate of the bandwidth of a simple LC resonator with a line impedance of $50 \Omega$ and a capacitance of $30 \mathrm{pF}\left(1 / 2 \pi Z_{0} C \approx 100 \mathrm{MHz}\right)$. The bandwidth in the parametrically amplified branch is a function of the parametric gain and thus a function of power [see Fig. 3(d)]. The gain-bandwidth product is constant in parametric amplifiers, ${ }^{9}$ corresponding to a lower bandwidth at higher parametric gain. At $100 \mathrm{mK}$, the line noise at $6.6 \mathrm{GHz}$ is quantum limited and a minimum parametric gain of 5 is needed to overcome the semiconductor amplifier noise. This value of gain corresponds to a bandwidth of approximately $20 \mathrm{MHz}$.

In conclusion, we have characterized a $\mathrm{Nb}$ scanning SQUID at $4 \mathrm{~K}$. Using homodyne detection to readout our dispersive RF SQUIDs, we reached a flux noise of $S_{\Phi}^{1 / 2}$ $=80 \mathrm{n} \Phi_{0} \mathrm{~Hz}^{-1 / 2}$ with $200 \mathrm{MHz}$ bandwidth at $4 \mathrm{~K}$. Furthermore, estimations predict that at lower temperature and higher excitation power, the flux noise decreases to 30 $\mathrm{n} \Phi_{0} \mathrm{~Hz}^{-1 / 2}$ at a bandwidth of $20 \mathrm{MHz}$. Thus, our microSQUID shows flux sensitivity on par with the best reported micro- and nano-SQUIDs, and features sub-micron pickup loops and integrated on-chip field coils and a scanning microscopy compatible geometry. A reduced back action and dissipation compared to conventional DC SQUIDs can be expected because of the lower operating frequency and easier variation of the excitation power. Furthermore, heating effects commonly found in on-chip resistors can be avoided. $^{20}$ These features make it ideal for single electron spin detection and manipulation with the goal of observing quantum coherent phenomena in mesoscopic samples.

This work was supported by the Alfried Krupp von Bohlen und Halbach Foundation and DFG Grant BL 1197/31. The authors thank D. Yohannes, O. Mukhanov, M. Radparvar, and A. Kirichenko from Hypres Inc. The SQUIDs used in this study were developed under an NSF IMR-MIP contract, Award No. 0957616.

${ }^{1}$ Y. Martin and H. K. Wickramasinghe, Appl. Phys. Lett. 50, 1455 (1987).

${ }^{2}$ A. M. Chang, H. D. Hallen, L. Harriott, H. F. Hess, H. L. Kao, J. Kwo, R. E. Miller, R. Wolfe, J. van der Ziel, and T. Y. Chang, Appl. Phys. Lett. 61, 1974 (1992).

${ }^{3}$ J. Kirtley and J. P. Wikswo, Annu. Rev. Mater. Sci. 29, 117 (1999).

${ }^{4}$ K. Hasselbach, C. Veauvy, and D. Mailly, Phys. C: Supercond. 332, 140 (2000).

${ }^{5}$ P. Maletinsky, S. Hong, M. S. Grinolds, B. Hausmann, M. D. Lukin, R. L. Walsworth, M. Loncar, and A. Yacoby, Nat. Nanotechnol. 7, 320 (2012). 
${ }^{6}$ C. C. Tsuei, J. R. Kirtley, C. C. Chi, L. S. Yu-Jahnes, A. Gupta, T. Shaw, J. Z. Sun, and M. B. Ketchen, Phys. Rev. Lett. 73, 593 (1994).

${ }^{7}$ H. Bluhm, N. C. Koshnick, J. A. Bert, M. E. Huber, and K. A. Moler, Phys. Rev. Lett. 102, 136802 (2009).

${ }^{8}$ K. C. Nowack, E. M. Spanton, M. Baenninger, M. Koenig, J. R. Kirtley, B. Kalisky, C. Ames, P. Leubner, C. Bruene, H. Buhmann, L. W. Molenkamp, D. Goldhaber-Gordon, and K. A. Moler, Nat. Mater. 12, 787 (2013).

${ }^{9}$ M. Hatridge, R. Vijay, D. H. Slichter, J. Clarke, and I. Siddiqi, Phys. Rev. B 83, 134501 (2011).

${ }^{10}$ E. M. Levenson-Falk, R. Vijay, N. Antler, and I. Siddiqi, Supercond. Sci. Technol. 26, 055015 (2013).

${ }^{11}$ M. A. Castellanos-Beltran, K. D. Irwin, G. C. Hilton, L. R. Vale, and K. W. Lehnert, Nat. Phys. 4, 929 (2008).

${ }^{12}$ R. Vijay, M. H. Devoret, and I. Siddiqi, Rev. Sci. Instrum. 80, 111101 (2009).

${ }^{13}$ K. O'Brien, C. Macklin, I. Siddiqi, and X. Zhang, Phys. Rev. Lett. 113, 157001 (2014).
${ }^{14}$ M. E. Huber, N. C. Koshnick, H. Bluhm, L. J. Archuleta, T. Azua, P. G. Bjoernsson, B. W. Gardner, S. T. Halloran, E. A. Lucero, and K. A. Moler, Rev. Sci. Instrum. 79, 053704 (2008).

${ }^{15}$ D. Vasyukov, Y. Anahory, L. Embon, D. Halbertal, J. Cuppens, L. Neeman, A. Finkler, Y. Segev, Y. Myasoedov, M. L. Rappaport, M. E. Huber, and E. Zeldov, Nat. Nanotechnol. 8, 639 (2013).

${ }^{16}$ N. C. Koshnick, M. E. Huber, J. A. Bert, C. W. Hicks, J. Large, H. Edwards, and K. A. Moler, Appl. Phys. Lett. 93, 243101 (2008).

${ }^{17}$ J. R. Kirtley, L. Paulius, A. J. Rosenberg, J. C. Palmstrom, C. M. Holland, E. M. Spanton, D. Schiessl, C. L. Jermain, J. Gibbons, Y.-K.-K. Fung, M. E. Huber, D. C. Ralph, M. B. Ketchen, G. W. Gibson, Jr., and K. A. Moler, Rev. Sci. Instrum. 87, 093702 (2016).

${ }^{18}$ The SQUID Handbook, edited by J. Clarke and A. J. Braginski (Wiley$\mathrm{VCH}, 2003)$

${ }^{19}$ C. D. Tesche and J. Clarke, J. Low Temp. Phys. 29, 301 (1977).

${ }^{20}$ F. C. Wellstood, C. Urbina, and J. Clarke, Phys. Rev. B 49, 5942 (1994). 